(iCB-NE-41?7

LBL-25769)

LBL -25769

DE89 013367

\title{
The Effect of Precipitation on Contaminant Dissolution and Transport: Analytic Solutions
}

W. B. Light, P. L. Chambre, T. H. Pigford, and W. W.-L. Lee

Department of Nuclear Engineering

University of California

and

Eanh Sciences Division

Lawrence Berkeley Laboratory

i Cyclotron Road

Berkeley, California 9.4720

September 1988

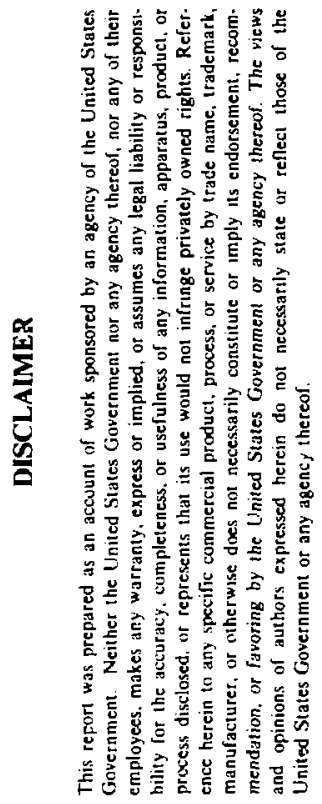

This work was supponed in part by the Manager, Chicago Operalions, Repository Technology and Transportation Program, ol the U.S. Department of Energy under Contract No. DE-AC0.3-76SForoys. 
The authors invite comments and would appreciate being notified of any errors in the report.

T. H. Pigford Department of Nuclear Engineering University of California Berkeley, CA 94720 


\section{Contents}

1. Introduction . . . . . . . . . . . . . . . . . . . . . . 1

2. Background . . . . . . . . . . . . . . . . . . . . . . . 1

3. Analysis . . . . . . . . . . . . . . . . . . . . . . . . 2

4. Numerical lllustrations . . . . . . . . . . . . . . . . . . . . . . . . . . . 10

5. Conclusions . . . . . . . . . . . . . . . . . . . . . . 11

References . . . . . . . . . . . . . . . . . . . . . 12

\section{Ist of Figures}

Figure 1. The stationary precipitation front.

Figure 2. Schematic of concentration profiles.

Figure 3. Dimensionless mass-transfer rate out from waste surface (upper curves) and precipitation front (lower curves) for $\alpha=5000, C_{o} / C_{p}=1000$.

Figure 4. Dimensionless mass-transfer rate out from waste surface (upper curves) and precipitation front (lower curves) for $\alpha=500, C_{o} / C_{p}=1000$.

Figure 5. Dimensionless mass-transfer tate out from waste surface (upper curves) and precipitation front (lower curves) for $\alpha=50, C_{o} / C_{p}=1000$. 


\section{Introduction}

Waste material in a geologic repository will eventually dissolve and migrate away for many want" inn ponents, this process will be lmited by the solubility of the waste matrix and species involved in the paper we deal with a single contamnant species and analyze the effect of precipitation caused by a ridured solubility of the contaminant at some distance from the waste package. The precipitation may be idi" t", local geochenical changes such as changes in temperature, pll or redox potential, caused by nearby geologle features or the waste itself.

If precipitation occurs it will be necessary to identify the key parameters in the precipitation process so that they can be studied in laboratory and field experiments. In addition to identifying the parameters that affect dissolution rate, this study shows how the precipitation process can be incorporated in the performance assessments of nuclear waste disposal.

If the U. S. Nuclear Regulatory Commission's release rate requirement ${ }^{1}$ for the engineered barrier system is to be met at the waste package/rock interface, precipitation can increase the mass transport rate near the waste package, and must be considered in showing compliance with the release rate criterion.

In contrast with other works on precipitation fronts, ${ }^{2,3.4 .5 .6}$ we provide analyti: solutions to the problem of precipitation at a stationary front. Numerical illustrations of these solutions are also presented.

\section{Background}

Garisto and Garisto ${ }^{2,4}$ and Garisto $0^{3}$ bave studied the effect of a stationary precipitation front near the waste on the dissolution rate of a spent fuel waste package. They predict enhanced dissolution rateg affecting not only the matrix but any congruently releaged contaminant as well. However, they did not state the rason dètre for a statinnary precipitation front.

One way that a atationary precipitation front can exist is by alpha radiolysis. Radioactive waste in contact with wacer may produce an excess of oxidation products by alpha radiolysib. If hydrogen gas escapes, the remaining radiolysis products, oxygen and bydrogen peroxide, can creatc a local oxidizing environment near the wasle. This would create a region of high redox potential around the waste where most actumde compounds show higher solubility than in the surrounding region of lower redox potential.

Neretnieks ${ }^{7}$ has postulated a redox front where hydrogen peroxide oxidizes ferrous iron from granitic rurk 
near the high-level nuclear waste. He estimates a front velocity of about $0.06 \mathrm{~mm} / \mathrm{yr}$ In a separate paper Neretnicks ${ }^{d}$ has considered the effect of such a moving redox front un uranium transport away from the oxidized region. He was not concerned with the effect on the rate of dissolution of the wasto itsilf Berause a nearby precipitation front can increase the net rate of dissolution of the solublity-lunted waste matrix, such as uranium in spent fuel, it can also increase the release rate of species that are released congruently with matrix dissolution.

This report presents an analytic solution to predict contaminant dissolution rates, as well as mass-transfer rates into the far field, based on transport by diffusion through a porous medium with spatially dependent contaminant solubility.

The analysis is formulated for a step-function reduction in the solubility from $C_{0}$ to $C_{p}$ at position $r_{p}$, but it is applicable to any system in which the solubility of a contaminant decreases with distance from the contaminant source and is constant with time.

The contaminant will dissolve from the waste surface and travel outward down the concentration gradient. At early times, concen* $n^{*}$ tions are quite low so that a solubility limit is not met in the porous rock away from the waste surface. Eventually, as the concentration increases, the solution may become locally saturated and precipitation will begin. The concentration will continue to rise in the region between the waste package surface and the precipitation front, finally reaching a steady-state profile.

Where there is local saturation, precipitate will form at a rate so as to balance the net rates of mass transfer into and out of the saturated region. For a sharp precipitation front, as postulated here, the saturated segion is reduced to a surface with zero thickness. In reality, the front could extend over a non-zero transition thickness. ${ }^{9}$ We also neglect the effect the accumulating precipitate might have on the transport process, such as filling the pores or moving as a colloid. The results presented here accurately describe the case of a sharp front and also provide a qualitative picture of systens wherein the solubility profile is a smooth function of distance.

\section{Anulyst:}

As illustrated in Figure 1, a sphi rical waste solid of radius $r_{0}$ is embedded in an infinite water-saturated porous medium. There is no contaminant in the porous medium when dissolution begins at time $t=0$, and direct contact is assumed between the waste and the porous medium (no container or other barriers). The solubility discontinuity, or precipitation front, is assumed to be a concentric spherical shell of radius 
$r_{p}$ surrounding the waste package (Figure 1). The dissolution rate at the waste surface is assumed to be governed by a linear solid-liquid reaction-rate expression with the forward reaction rate jo occurring when there is no contaminant in the pore waler and the minimum rate, zero, approached as the contammant concentration in the pore water approaches the solubility limit at the waste surface (see Finatwo (1)) In the linit when $j_{0}$ becomes sufficiently large, the concentration at the waste surfare quickly reaches the surface solubility $C_{\circ}$ and the expression reduces to that for a constant concentration boundary condition, C.

Transport in the porous medium is by fluid-phase diffusion only, with no advection Local sorption equilit. rium is assumed, and radioactive decay is neglected.

The precipitation front is assumed to be at a known, fixed location, $r_{p}$. At early times, when the concentration is below the solubility limit at the front location, the front has no effect on the transport process. The dornain from the waste surface to infinity is then treated as a single homogeneous region and we have

$$
\frac{\partial C_{1}}{\partial t}=\frac{D}{K} \frac{1}{r^{2}} \frac{\partial}{\partial r}\left(r^{2} \frac{\partial C_{1}}{\partial r}\right), \quad r_{0}<r<\infty, \quad 0<t<t_{p}
$$

where $C_{1}(r, t)$ is the fluid-phase contaminant concentration for $r_{0}<r<\infty$ and $0<t<t_{p}, D$ is the contaminant diffusion cuefficient in the pore fluid, $K$ is the retardation coefficient, and $t_{p}$ is the time at which the solubility limit is reached and precipitation begins. The initial concentration and the concentration at infinity are both assumed to be zero

$$
\begin{gathered}
C_{1}(r, 0)=0, \quad r_{0}<r<\infty \\
\lim _{r \rightarrow \infty} C_{1}(r, t)=0, \quad 0<i<t_{p}
\end{gathered}
$$

The dissolution rate at the waste surface is set equal to the assumed concentration-dependent reaction-ratc law

$$
-\left.c D \frac{\partial C_{1}}{\partial r}\right|_{r=r .}=\left.j \cdot\left(1-\frac{C_{1}}{C_{0}}\right)\right|_{r=r_{*} .} \quad 0<t<t_{p}
$$

where $c$ is the porosity and $j_{0}$ is the forward dissolution reaction rate per unit surface area of waste. To determine the precipitation time, $t_{p}$, we first solve (1)-(4). The contaminant concentration predicted by (1)-(4) increases steadily with time and monotonically decreases with distance from the waste (Figure 2 , $t<t_{p}$ ). Although it is possible that the conceatration may never reach the solubility limit anywhere other than the waste surface, if precipitation does occur we can determine $t_{p}$ implicitly from the equation

$$
C_{1}\left(r_{p}, t_{p}\right)=C_{p}
$$


For $t>t_{p}$ we divide the domain into two regions, one inside the front and the other outside the front. For the inner region

$$
\frac{\partial C_{2}}{\partial t}=\frac{D}{h} \frac{1}{r^{2}} \frac{\partial}{\partial r}\left(r^{2} \frac{\partial C_{2}}{\partial r}\right), \quad r_{0}<r<r_{p}, \quad t_{p}<t<\infty
$$

where $C_{2}(r, t)$ is the contaminant concentration for $r_{0}<r<r_{p}$ and $t_{p}<t<\infty$. The initial condition for this problem is given by the solution to (1)-(4) at $t=t_{\mathrm{p}}$

$$
C_{2}\left(r, t_{p}\right)=C_{1}\left(r, t_{p}\right), \quad r_{0}<r<r_{p}
$$

The boundary condition at the waste surface is the reaction-rate law as befcre, with $C_{2}$ replacing $C_{1}$

$$
-\left.c D \frac{\partial C_{2}}{\partial r}\right|_{r=r_{0}}=\left.j_{0}\left(1-\frac{C_{2}}{C_{0}}\right)\right|_{r=r_{0}, \quad} \quad t_{p}<t<\infty
$$

At the new boundary, $r=r_{p}$, we set the concentration to the solubility limit $C_{p}$

$$
C_{2}\left(r_{p}, t\right)=C_{p}, \quad t_{p}<t<\infty
$$

The region outside the precipitation front is treated similarly

$$
\frac{\partial C_{3}}{\partial t}=\frac{D}{K} \frac{1}{r^{2}} \frac{\partial}{\partial r}\left(r^{2} \frac{\partial C_{3}}{\partial r}\right), \quad r_{p}<r<\infty, \quad t_{p}<t<\infty
$$

with side conditions

$$
\begin{array}{cc}
C_{3}\left(r, t_{p}\right)=C_{1}\left(r, t_{p}\right), & r_{p}<r<\infty \\
C_{3}\left(r_{p}, t\right)=C_{p}, & t_{p}<t<\infty \\
\lim _{r \rightarrow \infty} C_{3}(r, t)=0, & t_{p}<t<m
\end{array}
$$

To bolve the equation system $(1)-(13)$ we first introduce the following dimensionless parameters

$$
\begin{array}{cl}
\rho \doteq r / r_{v}, & \rho_{p} \doteq r_{p} / r_{o} \\
\tau \doteq t D / K r_{* 1}^{2}, & \tau_{p} \doteq t_{p} D / K r_{o}^{2} \\
\alpha \doteq \frac{j_{o} r_{q}}{C D C_{0}}, & \alpha^{\prime} \doteq \alpha+1
\end{array}
$$

The problem is then simplified by introducing new dependent variables

$$
u_{1}(\rho, \tau) \doteq \rho C_{1}(\rho, \tau) / C_{0}, \quad u_{2}(\rho, \tau)=\rho C_{2}(\rho, \tau) / C_{o}, \quad u_{3}(\rho, \tau) \doteq \rho C_{3}(\rho, \tau) / C \text { o }
$$


The reformulated equations appear as the following system of equations (14)-(26)

$$
\begin{aligned}
& \frac{\partial u_{1}(\rho, \tau)}{\partial \tau}=\frac{\partial^{2} u_{1}(\rho, \tau)}{\partial \rho^{2}}, \quad 1<\rho<\infty, \quad 0<\tau<\tau_{p} \\
& u_{1}(\rho, 0)=0, \quad 1<\rho<\alpha \\
& -\left.\frac{\partial u_{1}(\rho, \tau)}{\partial \rho}\right|_{\rho=1}=\alpha-\alpha^{\prime} u_{1}(1, \tau), \quad 0<\tau<\tau_{p} \\
& \lim _{\rho \rightarrow \infty} \frac{u_{1}(\rho, \tau)}{\rho}=0, \quad 0<\tau<\tau_{p} \\
& u_{1}\left(\rho_{p}, \tau_{p}\right)=\rho_{p} C_{p} / C_{o} \\
& \frac{\partial u_{2}(\rho, \tau)}{\partial \tau}=\frac{\partial^{2} u_{2}(\rho, \tau)}{\partial \rho^{3}}, \quad 1<\rho<\rho_{p}, \quad \tau_{p}<\tau<\infty \\
& u_{2}\left(\rho, \tau_{p}\right)=u_{1}\left(\rho, \tau_{p}\right), \quad 1<\rho<\rho_{p} \\
& -\left.\frac{\partial u_{2}(\rho, \tau)}{\partial \rho}\right|_{f=1}=\alpha-\alpha^{\prime} u_{2}(1, \tau), \quad \tau_{p}<\tau<\infty \\
& u_{2}\left(\rho_{p}, \tau\right)=\rho_{p} C_{p} / C_{0}, \quad \tau_{p}<\tau<\infty \\
& \frac{\partial v_{3}(\rho, \tau)}{\partial \tau}=\frac{\partial^{2} u_{3}(\rho, \tau)}{\partial \rho^{2}}, \quad \rho_{p}<\rho<\infty, \quad \tau_{p}<r<\infty \\
& u_{3}\left(\rho, T_{p}\right)=u_{1}\left(\rho, \tau_{p}\right), \quad \rho_{p}<\rho<\infty \\
& u_{3}\left(\rho_{p}, \tau\right)=\rho_{p} C_{p} / C_{o}, \quad \tau_{p}<\tau<\infty \\
& \lim _{\rho \rightarrow \infty} \frac{u_{3}(\rho, \tau)}{\rho}=0, \quad \tau_{p}<\tau<\infty
\end{aligned}
$$

To solve (14)-(17), the Laplace tratrsform of (14) is taken with renpect to $t$ using the initial condition (15) with the result

$$
\Delta U_{1}(\rho, s)=\frac{\delta^{2} U_{1}(\rho, s)}{\partial \rho^{2}}, \quad 1<\rho<\infty
$$


where $U_{1}(\rho, s)$ is the Laplace transform of $u_{1}(\rho, \tau)$. The general solution to (27) taking into account boundary condition $(17)$ is

$$
U_{1}(\rho, s)=G(s) c^{-s} \cdot
$$

where $G(s)$ is an arbutrary function the transform of boundary condition (lii) is

$$
-\left.\frac{\partial L_{1}\left(\rho_{-} s\right)}{\partial \rho}\right|_{p=:}=\frac{\alpha}{s}-\alpha^{\prime} U_{1}^{\prime}(1, s)
$$

Substituting (28) into (29) determmes $G(6)$ and we get

$$
U_{1}(\rho, s)=\overline{s\left(\alpha^{\prime}+\sqrt{s}\right)} \exp (-(\rho-1) \sqrt{s}), \quad 1<\rho<\infty
$$

The inverse transform is given by Abramowitz and Stegun $(29.389)^{10}$

$$
u_{1}(\rho, \tau)=\frac{\alpha}{\alpha^{\prime}}\left[\operatorname{erc}\left(\frac{\rho-1}{2 \sqrt{\tau}}\right)-\exp \left(\alpha^{\prime}(\rho-1)+\alpha^{\prime 2} \gamma\right) \operatorname{erfc}\left(\alpha^{\prime} \sqrt{\tau}+\frac{\rho-1}{2 \sqrt{\tau}}\right)\right], \quad \begin{array}{ll}
1<\rho<\infty \\
& 0<\tau<\tau_{p}
\end{array}
$$

The final solution to (1)-(4) is then

$$
C_{1}(\rho, \tau)=\frac{C_{0}}{\rho} \frac{\alpha}{\alpha^{\prime}}\left[\operatorname{erfc}\left(\frac{\rho-1}{2 \sqrt{\tau}}\right)-\exp \left(\alpha^{\prime}(\rho-1)+\alpha^{\prime 2} \tau\right) \operatorname{erfc}\left(\alpha^{\prime} \sqrt{\tau}+\frac{\rho-1}{2 \sqrt{\tau}}\right)\right], \quad \begin{aligned}
& 1<\rho<\infty \\
& 0<\tau<\tau_{p}
\end{aligned}
$$

For the parameter values likely in a wet-rock repository ${ }^{2}, \alpha$ is much greater than unity so that $\alpha / \alpha^{\prime}$ is approximately equal to unity. Two of the three ratios $\rho_{p}, \tau_{p}$, and $C_{p} / C_{0}$ must be specified with the third determined by (18). We have chosen to specify the front location and the solubility ratio and to determine the precipitation time $\tau_{p}$ numerically using (18) and (31).

To solve (19)-(22), we assume a solution form $u_{2}(\rho, \tau)=v_{2}(\rho)+w_{2}(\rho, \tau)$ where $v_{2}(\rho)$ is the steady-state solution and $w_{3}(\rho, \tau)$ is the transient componeat of the given problem. The steady-state equations for $\nu_{2}(\rho)$ are those given by (19), (21) and (22) with the time derivative set equal to zero

$$
\begin{gathered}
v_{2}^{\prime \prime}(\rho)=0, \quad 1<\rho<\rho_{p} \\
-v_{2}^{\prime}(1)=\alpha-\alpha^{\prime} v_{2}(1) \\
v_{2}\left(\rho_{p}\right)=\rho_{p} C_{p} / C_{0}
\end{gathered}
$$

The solution to (33)-(35) is

$$
v_{2}(\rho)=a \rho+b, \quad 1<\rho<\rho_{p}
$$


with

$$
\begin{gathered}
a=\frac{\rho_{p}\left(\alpha^{\prime} / \alpha\right)\left(C_{p} / C_{0}\right)-1}{\rho_{p}\left(\alpha^{\prime} / \alpha\right)-1} \\
b=\frac{\rho_{p}\left(1-r_{p} / C_{u}\right)}{\rho_{p}\left(\alpha^{\prime} / \alpha\right)-1}
\end{gathered}
$$

We now solve the following problem for $\omega_{2}(\rho, \tau)$ so that the conbined solution $v_{2}(\rho)+w_{2}(\rho, \tau)$ satisfies the governing equations (19)-(22)

$$
\begin{gathered}
\frac{\partial w_{2}(\rho, \tau)}{\partial \tau}=\frac{\partial^{2} w_{2}(\rho, \tau)}{\partial \rho^{2}}, \quad 1<\rho<\rho_{p}, \quad \tau_{p}<\tau<\infty \\
w_{2}\left(\rho, \tau_{p}\right)=u_{1}\left(\rho, \tau_{p}\right)-v_{2}(\rho), \quad 1<\rho<\rho_{p} \\
-\left.\frac{\partial w_{2}(\rho, \tau)}{\partial \rho}\right|_{\rho=1}=-\alpha^{\prime} w_{2}(1, \tau), \quad \tau_{p}<\tau<\infty \\
w_{2}\left(\rho_{p}, \tau\right)=0, \quad \tau_{p}<\tau<\infty
\end{gathered}
$$

To salve (37)-(40). let $w_{2}(\rho, \tau)=\phi(\rho) \psi(\tau)$, then by $(37)$

$$
\psi^{\prime}(\tau)=-\lambda \psi(\tau)
$$

and

$$
\phi^{\prime \prime}(\rho)=-\lambda \phi(\rho)
$$

The boundary conditions (39) and (40) are applied to the new solution form to get

$$
\begin{gathered}
-\phi^{\prime}(1)=-\alpha^{\prime} \phi(1) \\
\phi\left(\rho_{p}\right)=0
\end{gathered}
$$

Equations (42)-(44) are solved to yield the eigeafunctions

$$
\phi_{n}(\rho)=\sin \left[\sqrt{\lambda_{n}}\left(\rho_{p}-\rho\right)\right], \quad 1<\rho<\rho_{p}
$$

witb the eigenvalues $\lambda_{n}$ determined by the solution to

$$
\tan \left[\sqrt{\lambda_{n}}\left(\rho_{p}-1\right)\right]=-\frac{\sqrt{\lambda_{n}}}{\alpha^{\prime}}
$$


The eigenvalues are deternined numericaily by intersectung the curves $y=\tan x$ and $y=-x / a^{\prime}\left(\rho_{p}-1\right)$ with one eigenvalue $\lambda_{n}=\left(x_{n} /\left(\rho_{p} .1\right)\right)^{2}$ for each intersection point $\left(x_{n}, y_{n}\right), x_{n}>0$ The rorresponding v $_{n}(\tau)$ function from (4l) is thosen to be

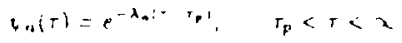

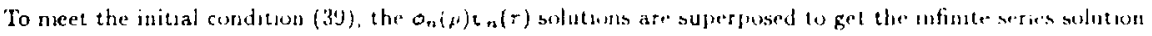

$$
u_{2}(\rho, \tau)=\sum_{n=1}^{\infty} A_{n} \psi_{n}(\rho) \boldsymbol{\psi}_{n}(\tau), \quad 1<\rho<\rho_{\rho}, \quad \tau_{r}<\tau<\infty
$$

where the series coeffic onts are given by

$$
A_{n}=\frac{1}{\left\|\phi_{n}\right\|^{2}} \int_{1}^{\rho} \phi_{n}(\rho)\left\{u_{1}\left(\rho, \tau_{p}\right)-v_{2}(\rho)\right] d \rho
$$

with the cigenfunction norm defined as

$$
\left\|\phi_{n}\right\|^{2} \doteq \int_{1}^{\prime \prime} \phi_{n}^{2}(\rho) d \rho
$$

The final solution to $(6)-(9)$ is then

$$
C_{2}(\rho, \tau)=C_{0}\left[a+\frac{b}{\rho}+\frac{1}{\rho} \sum_{n=1}^{\infty} A_{n} \phi_{n}(\rho) \psi_{n}(\tau)\right], \quad 1<\rho<\rho_{p}, \quad \tau_{,}<\tau<\infty
$$

To solve (23)-(26), we assume again the solution form $v_{3}(\rho, \tau)=v_{3}(\rho)+w_{3}(\rho, \tau)$ where $v_{3}(\rho)$, the steady-state solution, is to oatisfy

$$
\begin{gathered}
v_{3}^{\prime \prime}(\rho)=0, \quad \rho_{p}<\rho<\infty \\
v_{3}\left(\rho_{p}\right)=\rho_{p} C_{p} / C_{\rho} \\
\lim _{\rho \rightarrow \infty} \frac{v_{3}(\rho)}{\rho}=0
\end{gathered}
$$

and $w_{3}(\rho, \tau)$, the transient component, if to satisfy

$$
\begin{gathered}
\frac{\partial w_{3}(\rho, \tau)}{\partial \tau}=\frac{\partial^{2} w_{3}(\rho, \tau)}{\partial \rho^{2}}, \quad \rho_{\rho}<\rho<\infty, \quad \tau_{p}<\tau<\infty \\
w_{3}\left(\rho, \tau_{p}\right)=u_{1}\left(\rho, \tau_{p}\right)-v_{3}(\rho), \quad \rho_{p}<\rho<x
\end{gathered}
$$




$$
\begin{array}{cl}
w_{3}\left(\rho_{p}, \tau\right)=0, & \tau_{p}<\tau<\infty \\
\lim _{\rho \rightarrow \infty} \frac{w_{3}(\rho, \tau)}{\rho}=0, & \tau_{p}<\tau<\infty
\end{array}
$$

The solution to $(50)-(52)$ is

$$
\operatorname{trg}(\rho)=\rho_{p} C_{p} / C_{0}, \quad \rho_{p}<\rho<\infty
$$

To sol'. (53)-(56) we first shift the space-time boundaries by letting $x=\rho-\rho_{F}$ and $y=\tau-\tau_{p}$ to get the resulting new equations

$$
\begin{gathered}
\frac{\partial w_{3}(x, y)}{\partial y}=\frac{\partial^{2} w_{3}(x, y)}{\partial x^{2}}, \quad 0<x<\infty, \quad 0<y<\infty \\
w_{3}(x, 0)=u_{:}(x, 0)-v_{3}(x), \quad 0<x<\infty \\
u_{3}(0, y)=0, \quad 0<y<\infty \\
\lim _{x \rightarrow \infty} u_{3}(x, y)=0, \quad 0<y<\infty .
\end{gathered}
$$

The solution to (58)-(61) is given by

$$
w_{3}(x, y)=\int_{0}^{\infty}\left[u_{1}(\xi, 0)-v_{3}(\xi)\right] G^{*}(x, \xi, y) d \xi, \quad 0<x<\infty, \quad 0<y<\infty
$$

where the Green function $G^{*}$ is defined

$$
G^{*}(x, \xi, y)=\frac{1}{\sqrt{4 \pi y}}\left(\exp \left(\frac{-(x-\xi)^{2}}{4 y}\right)-\exp \left(\frac{-(x+\xi)^{2}}{4 y}\right)\right)
$$

Shifting back to $\rho$ and $\tau$ yields the result

$$
u_{3}(\rho, \tau)=\int_{\rho_{r}}^{\infty}\left[u_{1}\left(\eta, \tau_{p}\right)-v_{3}(\eta)\right]\left(\frac{\exp \left[\frac{-(p-\eta)^{2}}{4\left(\tau-\tau_{p}\right)}\right]-\exp \left[\frac{-\left(p+\eta-2 \rho_{p}\right)^{2}}{4\left(\tau-\tau_{p}\right)}\right]}{\sqrt{4 \pi\left(\tau-\tau_{p}\right)}}\right) d \eta, \quad \begin{array}{ll}
\rho_{p}<\rho<\infty \\
\tau_{p}<\tau<\infty
\end{array}
$$

The final solution to $(6)-(9)$ is then

$$
C_{3}(\rho, \tau)=\frac{\rho_{p} C_{p}}{\rho}+\frac{1}{\rho} \int_{\rho_{p}}^{\infty}\left\{\eta C_{1}\left(\eta, \tau_{p}\right)-\rho_{p} C_{p}\right]\left(\frac{\exp \left[\frac{-(\mu-\eta)^{2}}{4\left(\tau-\tau_{p}\right)}\right]-\exp \left[\frac{-\left(\rho, \eta-2 \rho_{p}\right)^{2}}{4(\tau-\tau p)}\right]}{\sqrt{4 \pi\left(\tau-\tau_{p}\right)}}\right) d \eta, \quad \begin{aligned}
& \rho_{p}<\rho<\infty \\
& \tau_{p}<\tau<\infty
\end{aligned}
$$


The mase-transfer rate, equal to the mass flux times the surface area of the spherical shell, is given by

$$
H_{1}(r, t)=-4 \pi r^{2} \in D \frac{\partial C_{c}(r, t)}{\partial r}, \quad i=1,2,3
$$

where $i=1,2$ or 3 depending on the space-time region of interest. The dimensionless mass-transfer rate is defined here to be

$$
\dot{m}_{1} \doteq \frac{\dot{M}}{4 \pi r_{0} c D C_{0}}, \quad i=1,2,3
$$

In dimensionless terms

$$
\dot{m}_{i}(\rho, \tau)=-\frac{\rho^{2}}{C_{0}} \frac{\partial C_{i}(\rho, \tau)}{\partial \rho}, \quad i=1,2,3
$$

Substituting $C_{i}(\rho, \tau)$ in for the three space-time domains yields, respectively

$$
\dot{m}_{1}(\rho, \tau)=\frac{\alpha}{\alpha^{\prime}} \operatorname{erfc}\left(\frac{\rho-1}{2 \sqrt{\tau}}\right)+\left(\alpha \rho-\frac{\alpha}{\alpha^{\prime}}\right) \exp \left(\frac{-(\rho-1)^{2}}{4 \tau}\right) \mathrm{F}\left(\frac{\rho-1}{2 \sqrt{\tau}}+\alpha^{\prime} \sqrt{\tau}\right), \quad \begin{array}{ll}
1<\rho<\infty \\
0<\tau<\tau_{\mathrm{F}}
\end{array}
$$

where

$$
\begin{aligned}
& F(x) \doteq \exp \left(x^{2}\right) \operatorname{erfc}(x) \\
& \dot{m}_{2}(\rho, \tau)=b+\sum_{n=1}^{\infty} A_{n}\left(\sin \left(\sqrt{\lambda_{n}}\left[\rho_{p}-\rho\right]\right)+\rho \sqrt{\lambda_{n}} \cos \left(\sqrt{\lambda_{n}}\left[\rho_{p}-\rho\right]\right)\right) \psi_{n}(\tau), \quad \begin{aligned}
& 1<\rho<\rho_{p} \\
& T_{p}<\tau<\infty
\end{aligned} \\
& \dot{m}_{3}(\rho, \tau)=\rho_{p} \frac{C_{p}}{C_{0}}+\int_{\rho_{p}}^{\infty} \frac{1}{C_{\mathrm{o}}}\left[\eta C_{1}\left(\eta, \tau_{p}\right)-p_{p} C_{\mathrm{r}}\right] \\
& \times\left(\frac{\left(1+\frac{\rho(\rho-\eta)}{2\left(\tau-\tau_{p}\right)}\right) \exp \left(\frac{-(\rho-\eta)^{2}}{4\left(r-\tau_{p}\right)}\right)-\left(1+\frac{d\left(p+\eta-2 \rho_{p}\right)}{2\left(\tau-\tau_{p}\right)}\right) \exp \left(\frac{-\left(\rho+\eta-2 \rho_{p}\right)^{2}}{\tau\left(\tau-\tau_{p}\right)}\right)}{\sqrt{4 \pi\left(\tau-\tau_{p}\right)}}\right) d \eta, \quad \begin{array}{ll}
\rho_{p}<\rho<\infty \\
\tau_{p}<\tau<\infty
\end{array}
\end{aligned}
$$

\section{Numertcal Ilustratlons}

Figure 3 shows the effect of a stationary precipitation front on the time-dependent dissolution rate and release rate of the solubility-limited contaminant ( $\mathrm{UO}_{2}$ in the case of opent nuclear fuel). The normalized masstrangfer rate at the waste surface (upper curvea), and the normalized mass-transfer rate outward from the precipitation front (lower curves) are plotted againgt dimensionless time with the location of the precipitaion front as a parameter. The start of precipitation, $\tau_{p}$, is indicated by the vertical bars.

The mass-transfer rates $\dot{M}[\mathrm{M} / \mathrm{t}]$ are normalized by the constant $4 \pi c r_{0} D C_{0}[\mathrm{M} / \mathrm{t}]$. Dimensionless time $\tau$ is obtained by dividing actual time by $K r_{0}^{2} / D[t]$. For the sample values $K=1000, r_{0}=0.5 \mathrm{~m}, D=10^{-5} \mathrm{~cm}^{2} / \mathrm{s}$, 
the time constant is about 8000 years. This means that the actual time scale on the figures wonld extend from about $10^{-2}$ to $10^{6}$ years. The mass-transfer normalization constant corresponds to the steady-state release rate from a waste sphere if no precipitation occurs away from the waste surface.

For this figure, the modified reaction-rate modulus $\left.\alpha=j_{0} r_{o} / c l\right) C_{o}$ has been set at 5000 . If the precipitation front is close to the waste surface, precipitation starts soon and steady state is reached early The mass. transfer rate at the waste surface at steady state is about 100 times higher for the precipitation front at $\left(r_{p}-r_{o}\right) / r_{0}=0.01$ than for the precipitation front at $\left(r_{p}-r_{0}\right) / r_{0}=10$ or for no precipitation front. If there is no precipitation front, the normalized steady-state rate at the waste surface is unity The precipitation front acts as a sink for dissolved contaminants. When it is located nearcr the waste it increases the concentration gradient and dissolution rate.

The mass-transfer rate leaving the front rises from zero at early time until the onset of precipitation at $\tau_{p}$, falling thereafter to the steady state value (in real dimensions) $4 \pi c r_{p} D C_{p}$. The steady-state mass release rate outside the precipitation front will be greater than that for no region of higher solubility by the ratio $\tau_{,} / r_{a}$

The mass-transfer rate into the front is the same by definition as the rate leaving the front for $t<t$, when there is no precipitation occurring. After the onset of precipitation, though not shown in Figure 3 , the rate into the front quickly rises to the same steady-state value as the steady-state mass-transfer rate frorn the waste, assuming no radioactive decay.

We see in Figure 3 that the steady-state rates leaving the front are many orders of magnitude less than the steady-state rates leaving the waste and entering the front. This indicates that nearly all the dissolved contaminant is precipitated and immobilized at the front.

As shown in Figure 4, a reduction of a to 500 reduces the early-time dissolution rate, when compared with Figure 3, due to the decrease in the rate of the forward dissolution reaction. The steady-state mass-transfer rate at the waste surface for $\left(r_{p}-r_{0}\right) / r_{0}=0.01$ is also slightly lower than in Figure?.

In Figure $5, \alpha$ is reduced still further to 50 , accompanied by additional lowering of the upper curves. The effect of reaction rate on the steady-state dissolution rate is more pronounced when the precipitation front is near the source. Thus, a precipitation front very near the waste surface can change what is otherwise a solubility-limited diffusion-controlled release rate to a relrase rate controlled by chemical reaction 


\section{b. Concluston}

In this report we have analysed the effect of precipitation on the dissolution and transport rales of a nondecaying contaminant. Precipitation near the waste surface can have a profound effect on dissolution and transport rates. At early times, the mass-Lransfer rate at the waste surface is controlled by the solid-liquid reaction rate to an extent determined by the modified reaction-rate modulus, a. At later times extending to steady state, the mass-transfer rate depends on the location of the precipitation front $\boldsymbol{r}_{p}$ and on the solubility ratio $C_{o} / C_{p}$. A precipitation front very near the waste surface can change the dissolution mechanism from solubility-diffusion-controlled to chemical-reaction-rate controlled.

Precipitation limits the concentration of the contaminant at $r>r_{p}$ to $C_{p}$, steepening the concentration gradient for dissolution on the aste parkage side of the front and flattening the gradient for transport in the region outside the front. This increases the rate of contaminant transport from the waste to the front while decreasing the rate of transport away from the front. when compared to the situation without precipitation. The difference in the transport rates at the front is the rate of precipitation. For large changes in solubility. most of the contaminant is immobilized by precipitation, as was observed in a parallel study.

The effect of a precipitation front lpcated nearby in surrounding rock is to increase the release rate at the waste surface/rock interface. The increase in release rate at the waste surface is greater the closer the precipitation and the laiger the ratio $C_{o} / C_{p}$, also cbserved by others. ${ }^{2,3,4,5,6}$ The release rates of other waste constituents that dissolve congruently with the solubility-controlling matrix can be increased by a local highsolubility region between the waste surface and the precipitation front. This study has identified the main parameters of precipitation that can affect release rates.

Although the analysis has been formulated here for a sharp precipitation front, the results can be applicable if the solubility decreases with distance from the waste surface in some known fashion. As auch this type of analysis may be applicable to tbe potential high-level nuclear waste repository. Precipitation may occur as contaminants leave hotter regions near the waste into cooler regions and it may occur if moisture penetrates the waste container, where there can be large spatial variations in oxidation potential.

\section{References}

I. U.S. NUCLEAR REGULATORY COMMISSION, Disposal of High-Level Radioactive Wastes in Geologic Repositories, 10 Code of Federal Regulations 60.113(a)(1)(ii)(B).

2. N. C. GARISTO \& F. GARISTO, "The Effect of Precipitation on the Long-Term Release of Radionuclides from Used Fuel," Ann. Nuc. Energy, IS, 591, (1986) 
3. F. GARISTO, "Solid Dissolution: Effect of Mass Transport-Precipitation Coupling." Chen Eng Sir. 11, 3219, (1986).

4. F. GARISTO \& N. C. GARISTO, "The Effect of Precipitation on Radionuclide Release from lsed Fucl," Proc. 2nd Intl Conf. on Radtoactuve Waste Management, 645, (1986).

5. N. C. GARISTO. K. B. HARUEY. F. GARSTO \& L. H. JOHNSON, "Source Trrm Modrls fert the" Assessment of Nuclear Fuel Waste Disposal in Canada." in Waste Agt. '86, 2, 397, (1986).

6. N. C. GARISTO \& F. GARJSTO, "Mass Transport-Precipitation Coupling in Finite Systents," Al:( L.- $^{-}$ $9562(1988)$.

7. I. NERETNIEKS, "The Movement of a Redox Front Downsi ream From A Repository For Nuclear Wiaste". Nuclear Technology, 62, 110, (1983).

8. 1. NERETNIEKS \& B. ASLUND, "The Movement of Radionuclides Past a Redox Front", KBS "l'll 83-66i. (1986).

9. P. L. CHAMBRE, Unpublished (1987).

10. M. ABRAMOWITZ \& I. A. STEGUN (eds.), Handbook of Mathematical Functions. New York, Dover, (1965). 


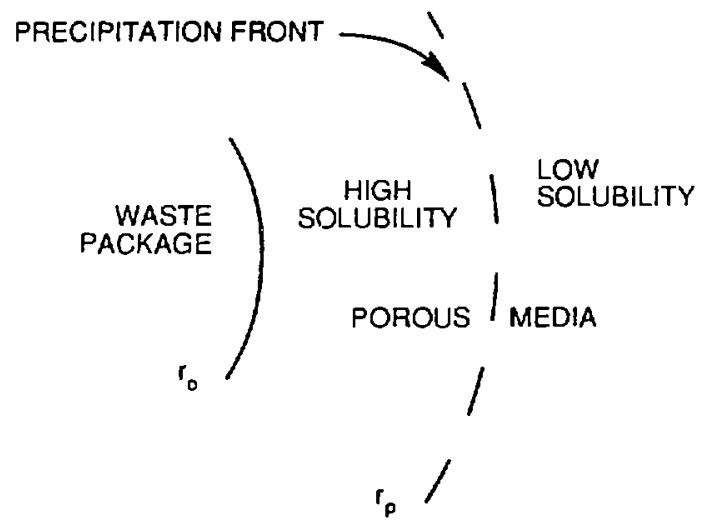

Figure 1. The stationary precipitation front.

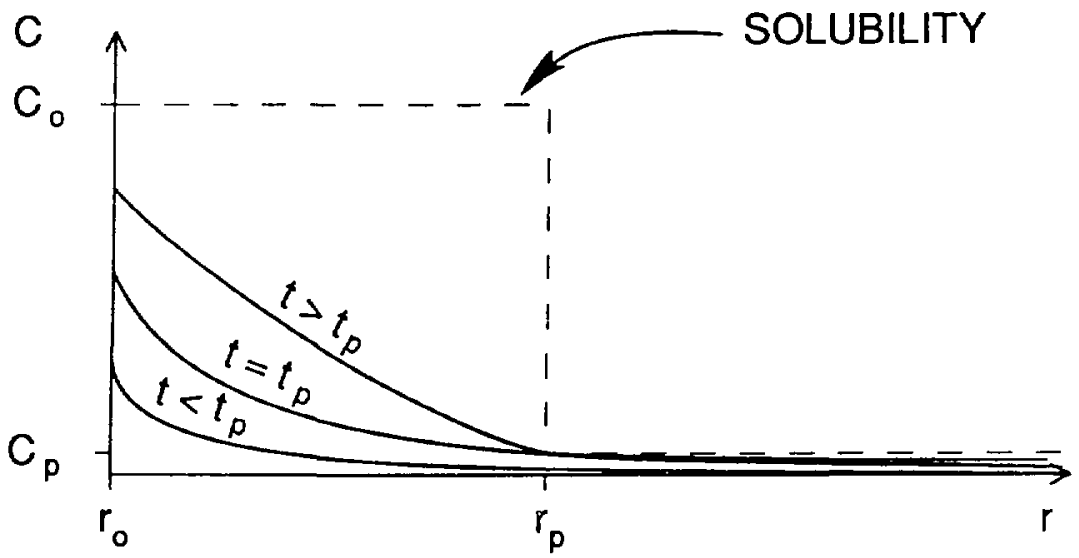

Figure 2. Schematic of concentration profiles. 


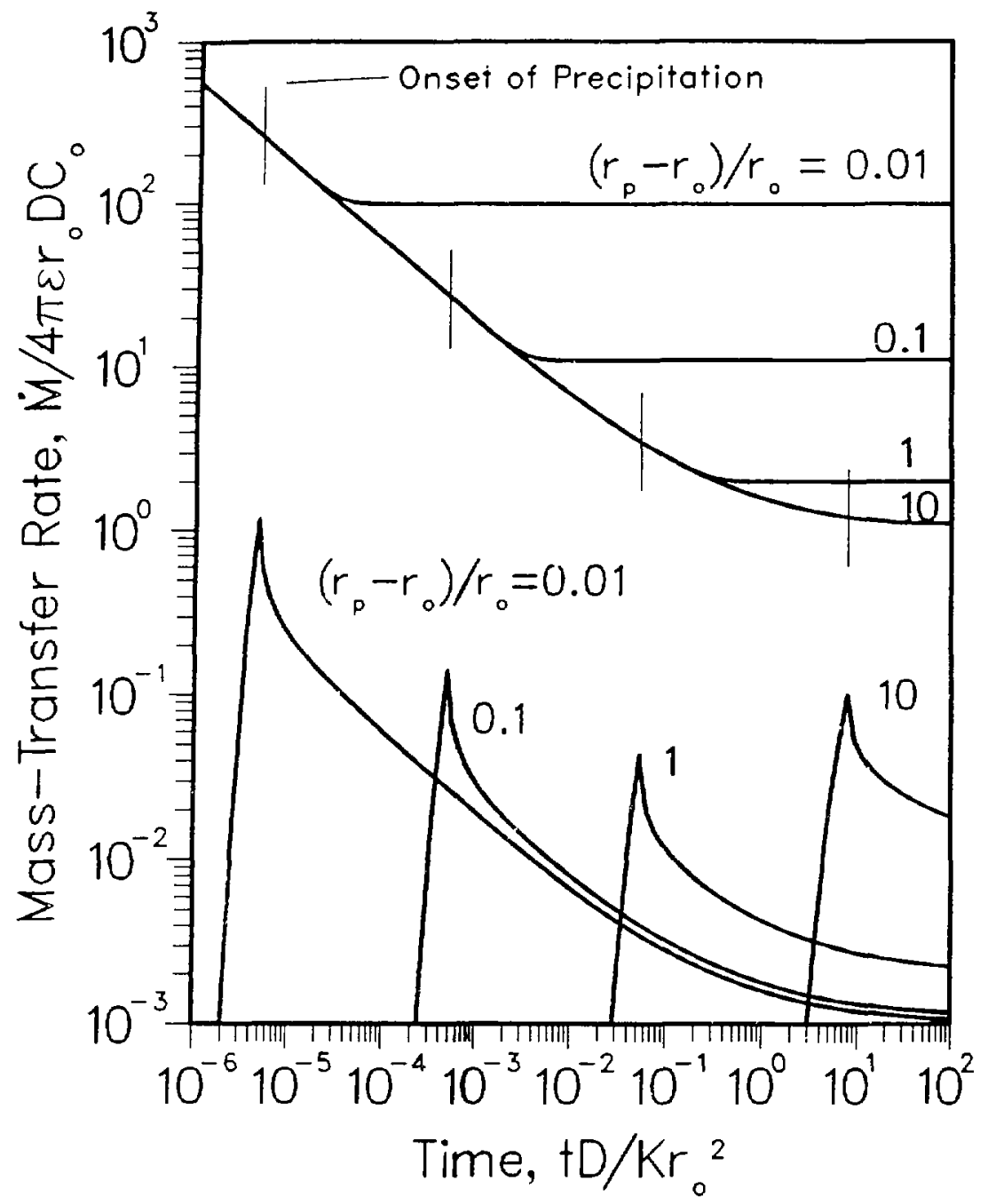

Figure 3. Dimensionless mass-transfer rate out from waste surface (upper curves) and precipitation front (lower curves) for $\alpha=5000, C_{0} / C_{p}=1000$. 


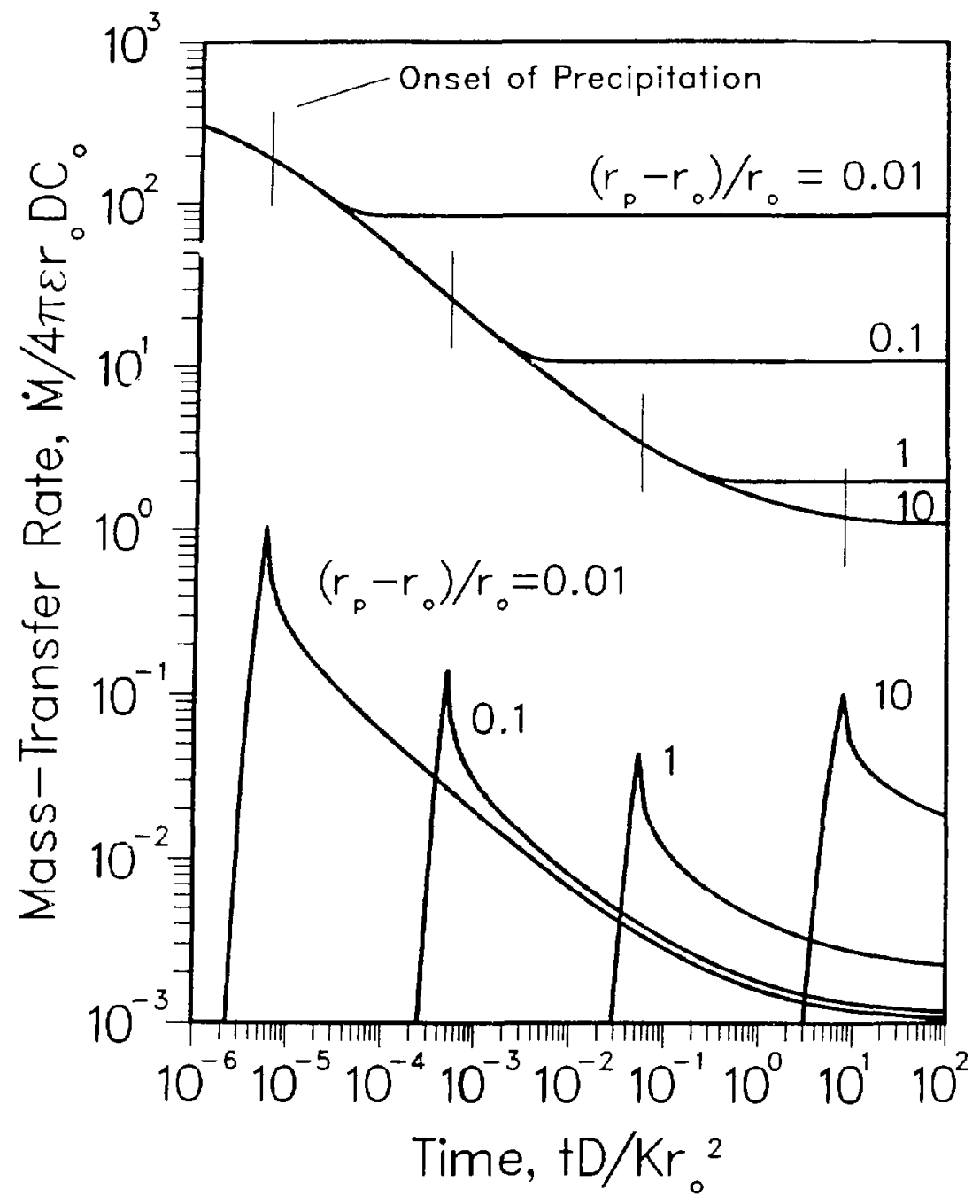

Figure 4. Dimensionless mass-transfer rate out from waste surface (upper curves) and precipitation front (lower curves) for $\alpha=500, C_{0} / C_{p}=1000$. 


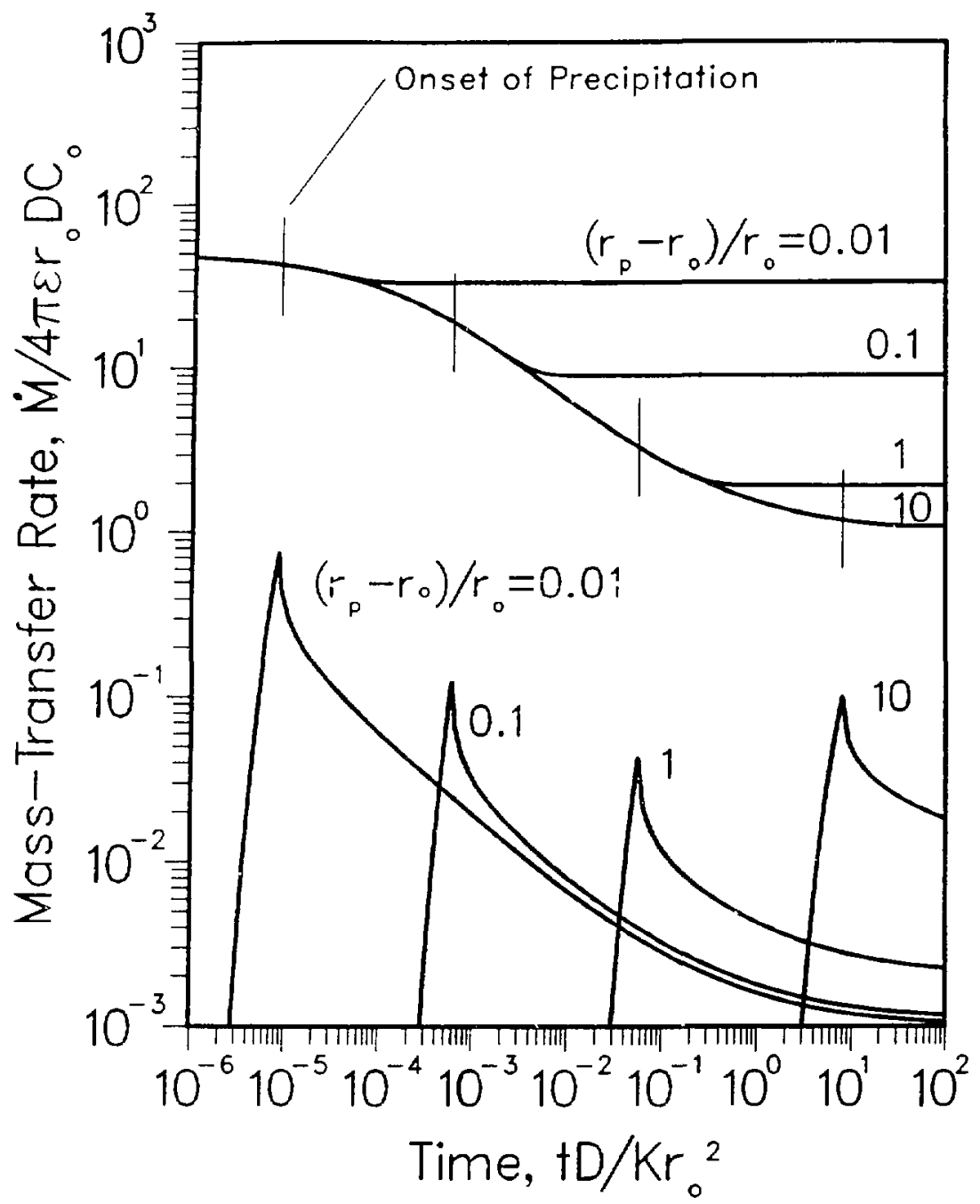

Figure 5. Dimensionless mass-transfer rate out from waste surface (upper curves) and precipitation front (lower curves) for $\alpha=50, C_{0} / C_{p}=1000$. 